\title{
Ustekinumab exposure-outcome analysis in Crohn's disease only in part explains limited endoscopic remission rates
}

Bram Verstockt, $M D^{1,2}$, Erwin Dreesen, PharmD $^{3}$, Maja Noman, $M D^{1}$, An Outtier, $M D^{1}$, Nathalie Van den Berghe ${ }^{3}$, Isolde Aerden ${ }^{1}$, Griet Compernolle ${ }^{3}$, Gert Van Assche, MD, $\mathrm{PhD}^{1,2}$, Ann Gils, PharmD, $\mathrm{PhD}^{3}$, Séverine Vermeire, $\mathrm{MD}, \mathrm{PhD}^{1,2}$, Marc Ferrante, $\mathrm{MD}, \mathrm{PhD}^{1,2}$

1. Department of Gastroenterology and Hepatology, University Hospitals Leuven, KU Leuven, Leuven, Belgium.

2. Department of Chronic Diseases, Metabolism and Ageing, KU Leuven, Leuven, Belgium.

3. Department of Pharmaceutical and Pharmacological Sciences, KU Leuven, Leuven, Belgium.

Running title: Ustekinumab dose-response relationship in real-life

Corresponding author: Prof. Dr. Marc Ferrante Dpt. Gastroenterology and Hepatology University Hospitals Leuven, KU Leuven Herestraat 49 B-3000 Leuven, BELGIUM marc.ferrante@uzleuven.be Tel 016344225 Fax 016344419

Copyright (C 2019 European Crohn's and Colitis Organisation (ECCO). Published by Oxford University Press. All rights reserved. For permissions, please email: journals.permissions@oup.com 


\section{Abstract}

\section{Background and aims}

Ustekinumab, an anti-IL12/23p40 monoclonal antibody, has been approved for Crohn's disease (CD). Real-life data in CD patients receiving ustekinumab intravenously (IV) during induction followed by subcutaneous (SC) maintenance are lacking. We assessed efficacy of ustekinumab and studied exposure-response correlations.

\section{Methods}

We performed a prospective study in 86 CD patients predominantly refractory or intolerant to anti-tumour necrosis factor agents and/or vedolizumab. All received ustekinumab $6 \mathrm{mg} / \mathrm{kg}$ IV induction, with 90mg SC q8w thereafter. Endoscopic response (50\% decrease in Simple Endoscopic Score for CD (SES-CD) at week 24), endoscopic remission (SES-CD $\leq 2$ ), and clinical remission (daily stool frequency $\leq 2.8$ and abdominal pain score $\leq 1$ ) were assessed at week 4,8,16 and 24. Further serial analyses included patient reported outcomes (PRO2), faecal calprotectin (fCal) and ustekinumab serum levels.

\section{Results}

SES-CD decreased from $11.5(8.0-18.0)$ at baseline to $9.0(6.0-16.0)$ at w24 $(p=0.0009)$, but proportions of patients achieving endoscopic response (20.5\%) or endoscopic remission (7.1\%) were low. Clinical remission rates were $39.5 \%$ at w24. After IV induction, fCal dropped from baseline $(1242.9 \mu \mathrm{g} / \mathrm{g})$ to $w 4(529.0 \mu \mathrm{g} / \mathrm{g})$ and w8 $(372.2 \mu \mathrm{g} / \mathrm{g})$, but increased again by w16 $(537.4 \mu \mathrm{g} / \mathrm{g})$ and w24 $(749.0 \mu \mathrm{g} / \mathrm{g})$. A clear exposure-response relationship was observed, both during induction as during maintenance therapy, with different thresholds depending on the targeted outcome. 


\section{Conclusion}

In this cohort of refractory CD patients, ustekinumab showed good clinical remission rates, but limited endoscopic remission after 24 weeks. Our data suggest that higher doses may be required to achieve better endoscopic outcomes.

Key words: ustekinumab, real-life, calprotectin, endoscopic remission, therapeutic drug monitoring, Crohn's disease 


\section{ABBREVIATIONS}

CADTH Canadian Agency for Drugs and Technologies in Health

CD Crohn's disease

$\mathrm{Cl} \quad$ confidence interval

CRP C-reactive protein

ECCO European Crohn's and Colitis Organisation

EMA European Medicines Agency

fCAL faecal calprotectin

FDA Food and Drug Administration

IBD inflammatory bowel disease

IV intravenous

NPV negative predictive value

NSAIDS non-steroidal anti-inflammatory drugs

PPV positive predictive value

ROC area under the receiving operating characteristic curve

SC subcutaneous

TDM therapeutic drug monitoring

TNF tumour necrosis factor 


\section{INTRODUCTION}

The treatment of patients with Crohn's disease (CD) entirely changed over the past two decades. With the arrival of anti-tumour necrosis factor alpha (anti-TNF) therapy, therapeutic targets evolved beyond clinical remission, ultimately aiming mucosal healing. ${ }^{1}$ However, anti-TNF induced endoscopic remission rates vary from $13.1 \%$ to $44 \%$ and are therefore far from ideal. ${ }^{2}$ Fortunately, new treatment options have been actively explored and already entered clinical practice. ${ }^{3}$

Ustekinumab (Janssen Biotech, Inc.), a fully human IgG1 monoclonal antibody, targets the IL-12/IL-23 shared p40 subunit. ${ }^{4}$ Clinical efficacy was primarily proven in psoriasis and psoriatic arthritis, ${ }^{5-7}$ whereafter it was studied in CD. $^{8}$ The UNITI program clearly demonstrated the potency of ustekinumab to induce and maintain clinical remission in moderate-to-severe CD. ${ }^{9}$ resulting in the approval by Food and Drug Administration (FDA), European Medicines Agency (EMA) and the Canadian Agency for Drugs and Technologies in Health (CADTH). However, real-life data in $C D$ patients receiving ustekinumab intravenously (IV) during induction followed by a subcutaneous (SC) maintenance regimen are currently lacking, as well as data on endoscopic remission and biomarkers such as faecal calprotectin (fCal). ${ }^{10-14}$

All biologics have the potential of inducing immunogenicity, resulting in decreased drug levels and loss of response. In the context of anti-TNF therapy, a dose-response relationship has been established, ${ }^{15}$ leading to the recommended approach of reactive therapeutic drug monitoring (TDM). ${ }^{16}$ In the UNITI program, serum concentrations of ustekinumab were proportional to dose, and associated with clinical efficacy. ${ }^{17}$ Also limited real-life data suggested better outcomes with higher exposure,,$^{10,14,18}$ though this could not be validated by others. ${ }^{19}$ Nevertheless, due to the different assays used to measure ustekinumab serum 
levels and due to the lack of standardized dosing and endoscopic assessment, the ideal therapeutic window to aim for is still debated.

We therefore conducted this single centre, prospective open-label cohort study to assess clinical and endoscopic outcomes at predefined time points and to explore the kinetics of biomarkers faecal calprotectin and C-reactive protein (CRP). Finally, we also studied exposure-response relationships.

\section{MATERIALS AND METHODS}

\section{Study design and patients}

We conducted this prospective, open-label cohort study at the University Hospitals Leuven (Leuven, Belgium). All patients included in the analysis had given written consent to participate in the Institutional Review Board approved IBD Biobank (B322201213950/S53684), whereby serum, faeces, biopsies, and clinical characteristics are collected prospectively on serial and predefined time points.

All consecutive CD patients who initiated ustekinumab between September 2016 and January 2018 were screened, allowing a follow-up of at least 24 weeks for every patient. Patients with an ostomy or without active endoscopic or radiographic disease at baseline were excluded. Similarly, patients with a diagnosis of IBD unclassified, as well CD patients with pure perianal involvement without luminal disease were excluded. None of our patients had symptomatic strictures or known intra-abdominal fistulas at the time of ustekinumab initiation. Finally, patients who were referred back to their treating gastroenterologist after 
having received their IV induction at our unit were excluded from the analysis, due to lack of adequate prospective follow-up data.

All included patients received an ustekinumab loading-dose of approximately $6 \mathrm{mg}$ per kilogram bodyweight (520 mg for patients with body weight above $85 \mathrm{~kg}$, $390 \mathrm{mg}$ between $65-85 \mathrm{~kg}$ and $260 \mathrm{mg}$ below $65 \mathrm{~kg}$ ) at week 0 (w0), followed by a fixed maintenance dose of 90mg SC every eight weeks. Latent tuberculosis was excluded via chest X-ray and tuberculin skin test or interferon gamma release assay in all patients, prior to first ustekinumab administration. Serum samples were prospectively collected during induction (w0, w4 and w8) and during maintenance (w16 and w24), and stored at $-20^{\circ} \mathrm{C}$. Apart from w4 (intermediate time point), serum samples were collected at trough (prior to the following $\mathrm{SC}$ injection).

\section{Outcomes}

A weighted patient-reported outcome (PRO2) was calculated at week 4, week 8, week 16 and week 24 , and defined as $5 x$ abdominal pain score $+2 x$ liquid stool frequency. ${ }^{20}$ Clinical remission, defined as an average daily stool frequency $\leq 2.8$ and an average abdominal pain score $\leq 1 .{ }^{21}$ Biological remission was defined as a CRP $\leq 5 \mathrm{mg} / \mathrm{L}$ and response as either $\mathrm{a} \geq$ $50 \%$ decrease in CRP or a CRP $\leq 5 \mathrm{mg} / \mathrm{L}$, in patients with an elevated CRP at baseline (CRP $>5 \mathrm{mg} / \mathrm{L})$. The primary endpoint of this study, endoscopic remission, was assessed at week 24 in all patients, as suggested by recent ECCO guidelines, ${ }^{22}$ and defined as a Simple Endoscopic Score for CD (SES-CD) $\leq 2 .{ }^{23,24}$ All endoscopies were performed by the same 3 experienced IBD staff members (GVA, SV, MF), and the SES-CD score was filled out prospectively in the electronic medical record. Endoscopic response was considered in case of a minimal $50 \%$ SES-CD decrease compared to baseline. ${ }^{23,24}$ Endoscopic improvement was defined as a minimal decrease of 3 points in the SES-CD score compared to baseline. ${ }^{24}$ 
For 4 patients with pure small intestinal involvement, response was also assessed by a dedicated abdominal radiologist using magnetic resonance enterography. All response and remission rates were analysed as intention-to-treat.

\section{Biomarkers}

Faecal calprotectin. Faecal samples were collected at home in all patients (at baseline, w4, w8, w16 and w24), stored at $4^{\circ} \mathrm{C}$ in the home fridge and transported cooled within 24 hours to the hospital. fCal measurements were performed for all patients with the fCAL ELISA kit (Bühlmann, Schönenbuch, Switzerland).

C-reactive protein. CRP was determined by the routine laboratory of the University Hospitals Leuven and measured at every visit in all patients (at baseline, w4, w8, w16 and w24).

\section{Measurements}

Ustekinumab serum concentrations. Ustekinumab serum concentrations were determined by use of an in-house developed sandwich-type enzyme-linked immunosorbent assay. In the assay, ustekinumab is captured between MA-UST56A2D11 as coating antibody and biotinylated MA-UST56C1H12 as capture antibody, allowing the detection of ustekinumab concentrations ranging from $0.25 \mu \mathrm{g} / \mathrm{ml}$ to $64.0 \mu \mathrm{g} / \mathrm{ml}$. This in-house developed ustekinumab assay has been shown to be comparable to the ustekinumab assay of Janssen R\&D in terms of specificity, selectivity, accuracy and precision. ${ }^{25}$ Serum concentrations were measured in all patients at every visit (at baseline, w4, w8, w16 and w24). 
Ustekinumab antibodies. A drug-sensitive bridging assay using ustekinumab as both coating and capture antibody and MA-UST37F12 as calibrator was used to quantify antiustekinumab antibodies. Anti-ustekinumab levels above a cut-off of $5.0 \mathrm{ng} / \mathrm{ml}$ equivalents MA-UST37F12 were considered positive. This drug-sensitive assay allows the quantification of unbound anti-ustekinumab antibodies in samples with ustekinumab concentrations below the lower limit of quantification. Additionally, anti-ustekinumab antibodies were measured using a drug-tolerant affinity capture elution assay, similarly developed as the anti-infliximab assay. ${ }^{26}$ Antibodies were measured in all samples with an ustekinumab serum level < $1.0 \mu \mathrm{g} / \mathrm{mL}$. 


\section{Statistical Analysis}

All analyses were carried out using $\mathrm{R}$ version 3.5.0 (R Development Core Team, Vienna, Austria). A non-responder imputation was applied, as well as a last-observation carried forward approach in patients discontinuing therapy prior to the primary endpoint at week 24 . Continuous variables are expressed as median and interquartile range (IQR). Unpaired data were compared using the Mann-Whitney U-test for continuous variables, and Fisher's exact or Chi-Square test for categorical variables. Continuous paired data were compared using the Wilcoxon signed-rank test. All data below the limit of quantification but above limit of detection were substituted with the value of the lower limit of quantification, i.e., $0.25 \mu \mathrm{g} / \mathrm{mL}$ for ustekinumab serum concentrations, $0.3 \mathrm{mg} / \mathrm{L}$ for CRP and $30 \mu \mathrm{g} / \mathrm{g}$ for fCal. Time to event data (i.e., time to treatment discontinuation) were analysed using the Kaplan-Meier method. Diagnostic performance was assessed with receiver operating characteristics (ROC) curve analysis. A clinically relevant threshold value was chosen on the ROC curve, based on the performance of the Youden's J statistic or the closest top-left method. Stepwise forward addition-backward elimination binary logistic and linear regression modelling was performed to identify independent predictors of outcome and drug exposure. Final model selection was based on the most optimal second-order Akaike information criterion. A two-tailed p-value $<0.05$ was considered significant.

\section{RESULTS}

\section{Patient characteristics}

From September 2016 till January 2018, a total of 118 CD patients who initiated ustekinumab therapy at our centre were screened, of whom 86 CD (58 women, 67.4\%) patients with active endoscopic and/or radiologic disease met the inclusion and exclusion 
criteria (Supplementary Figure 1). Baseline demographic and clinical characteristics are summarized in Table 1. With a median disease duration of 14.7 (interquartile range, IQR 6.0-23.3) years at initiation of ustekinumab, the vast majority of patients had previously been exposed to anti-TNF or vedolizumab therapy (95.3\%, 67.4\% respectively).

\section{Treatment response}

\section{Endoscopic remission}

SES-CD scores decreased significantly from baseline (median 11.5, IQR 8.0-18.0) to week 24 (median 9.0, IQR 6.0-16.0) ( $\mathrm{p}=0.0009)$ (Figure 1). Nevertheless, endoscopic response was observed in only $20.5 \%$ of patients, and endoscopic remission achieved in no more than 6 patients $(7.1 \%)$. In the very small subgroup $(n=4)$ of biologically naive patients, only one patient achieved endoscopic response, but none endoscopic remission. Using binomial generalized linear models, we studied the probability of a successful endoscopic response to ustekinumab. Being female and a minimal $50 \%$ drop in faecal calprotectin from baseline to week 8 were independent predictors of endoscopic response at week 24 . No significant predictors of endoscopic remission could be identified (Supplementary Table 1).

\section{Effect of ustekinumab on biomarkers}

Among the 60 patients with an elevated CRP at baseline (69.8\%), a decrease in CRP was observed compared to baseline (Figure 2A), resulting in biological response and remission rates of $55.0 \%$ and $40.0 \%$ by week 8 , and $53.3 \%$ and $31.7 \%$ by week 24 , respectively.

Faecal calprotectin significantly decreased from baseline (median 1242.3, IQR 470.51800.0) to week 4 (median 529.0, IQR, 182.2-1780.8, $\mathrm{p}=0.0008$ ), further down to week 8 (median 372.2, IQR, 137.0-1518.7, $\mathrm{p}=5.2 \times 10^{-5}$ compared to baseline). By week $8,43.7 \%$ of 
patients had a minimal $50 \%$ decrease in fCal. However, fCal increased again by week 16 (median 537.4, IQR, 167.0-1741.2, $\mathrm{p}=0.001$ compared to baseline) and even further by week 24, resulting in a fCal level after 6 months approaching again baseline fCal levels (median 749.0, IQR, 184.5-1800.0, p=0.03 compared to baseline) (Figure 2B). Out of all patients with elevated CRP at baseline, $30.0 \%$ experienced a 50\% decrease in both CRP and fCal by week 8 , whereas $32.1 \%$ had a CRP $<5 \mathrm{mg} / \mathrm{dL}$ and a fCal $<250 \mu \mathrm{g} / \mathrm{g}$ at week 24 .

Remarkably, fCal initially decreased in both future endoscopic responders as nonresponders. Although it further decreased in responders, it started to increase again beyond week 8 in non-responders (Supplementary Figure 2). Patients who did not reach a 50\% decrease in $\mathrm{fCal}$ by week 8 , had a significant higher chance to discontinue therapy later on (odds ratio 4.0, 95\% confidence interval, $\mathrm{Cl} 1.5-11.2, \mathrm{p}=0.006$ ).

\section{Clinical remission}

The IV administration of ustekinumab therapy induced a significant decrease in the median weighted PRO2 score from baseline to week $8\left(p=8.9 \times 10^{-7}\right)$, with a week 8 clinical remission and steroid-free clinical remission rate of $36.0 \%$ and $31.4 \%$ respectively. By week $24,39.5 \%$ and $38.4 \%$ of patients achieved clinical remission and steroid-free clinical remission, respectively. Lower baseline fCal $(p=0.03)$, lower baseline CRP $(p=0.05)$ and biological remission by week $8(p=0.05)$ were withheld as predictors for clinical remission by week 24 . From the clinical remitters at week $8,71.9 \%$ reported sustained clinical remission at week 24. In contrast, $21.8 \%$ of those not in clinical remission by week $8(n=55)$ were in clinical remission by week 24 . 
Patients were followed up for a median of $32.0(24.0-46.3)$ weeks, with $33(38.4 \%)$ patients discontinuing therapy after a median of $24.0(22.0-29.0)$ weeks. The main reason for ustekinumab discontinuation was the absence of an objective improvement after 6 months of therapy $(n=31,93.9 \%)$, with $(n=3)$ or without $(n=28)$ adverse events. Two patients $(6.1 \%)$ discontinued therapy only because of side-effects.

\section{Ustekinumab serum concentrations}

Median (IQR) ustekinumab serum concentration were $21.3 \mu \mathrm{g} / \mathrm{mL}(13.4-25.2)$ at week 4 , and $7.2 \mu \mathrm{g} / \mathrm{mL}$ (3.4-10.5) at week 8 (Supplementary Figure 3). During SC maintenance, median (IQR) serum levels were $2.6 \mu \mathrm{g} / \mathrm{mL}(1.1-4.2)$ at week 16 and $2.1 \mu \mathrm{g} / \mathrm{mL}(0.8-3.2)$ at week 24 . Only $1.6 \%$ of all week 24 samples had a trough concentration above the previously suggested level of $4.5 \mu \mathrm{g} / \mathrm{mL} .{ }^{14}$ A higher baseline serum albumin, a lower baseline fCal and female sex were shown to be independent predictors of higher ustekinumab serum concentrations during induction. Prior exposure to anti-TNF agents negatively influenced maintenance levels, with further decreasing levels the more anti-TNF agents the patient was previously exposed to (Supplementary Table 2). At all time points, ustekinumab serum levels and faecal calprotectin were significantly, inversely correlated (Supplementary Table 3).

\section{Immunogenicity}

Anti-ustekinumab antibodies were measured in all samples with an ustekinumab level below $1 \mu \mathrm{g} / \mathrm{mL}(\mathrm{n}=57)$. Only one patient had detectable anti-ustekinumab antibodies: using a drugsensitive assay both at week 8 and week 16, however with a drug-tolerant assay already by week 4. 


\section{An exposure-response relationship}

Quartile analysis clearly demonstrated that fewer patients with an ustekinumab serum concentration in the lowest quartile (Q1) at week 4 and week 8, experienced a $50 \%$ decrease in fCal by week $8(p=0.008, p<0.001$ respectively) (Figure 3). Similar trends could be observed between week 4 , week 8 , week 16 or week 24 serum levels and endoscopic response or endoscopic remission after 6 months (Supplementary Figure 4 - 5). Numerically, ustekinumab serum concentrations were higher in endoscopic responders at every time point (Figure 4, Table 2).

At week 8 , a minimal ustekinumab exposure of $4.2 \mu \mathrm{g} / \mathrm{mL}$ was needed to achieve a $50 \%$ decrease in fCal (NPV 87.0\%, $p=0.004)$. Although not significant, $(p=0.25)$ a serum level below $5.0 \mu \mathrm{g} / \mathrm{mL}$ at week 8 almost ruled out endoscopic response later on (NPV $90.0 \%$ ) (Table 2, Supplementary Table 4). During maintenance, thresholds of $2.3 \mu \mathrm{g} / \mathrm{mL}$ (week 16) and $1.9 \mu \mathrm{g} / \mathrm{mL}$ (week 24) were identified as the minimal exposure needed to maximize the likelihood of endoscopic response after 6 months (NPV $87.9 \%$ and $85.2 \%$ respectively) (Table 2). Due to the low endoscopic remission rates, no statistically relevant analyses could be performed to identify the ideal cut-off for endoscopic remission.

\section{Safety profile}

No new safety concerns arose during this observational cohort study. Apart from known mild side-effects including arthralgia and upper respiratory tract infections, ${ }^{27}$ two patients reported a pneumonia and one suffered from an abscess in the right kidney. Two patients had a non-disseminated herpes zoster infection. One patient with home parenteral nutrition, suffered from a catheter sepsis. One patient developed urticaria during IV induction, which further increased the day after with shortness of breath and swollen lips, and then 
spontaneously disappeared. Detailed allergological investigations (including ustekinumab skin tests) excluded an IgE mediated allergy or delayed hypersensitivity, but diagnosed a non-steroidal anti-inflammatory drug-induced angioedema. No ustekinumab-antibodies were found in this patient. Furthermore, the patient could continue ustekinumab therapy without recurrence of the reaction. Finally, one patient who was earlier diagnosed with a small fiber neuropathy of unknown origin, reported increasing symptoms after ustekinumab IV induction.

Overall, five patients discontinued therapy because of adverse events (with or without efficacy): two patients suffered from debilitating arthralgia, one patient reported atypical symptoms including nausea and fatigue, one patient had vague complaints of itching, shortness of breath and fatigue after SC administration, and one patient reported general malaise after initiating ustekinumab. Interestingly, the latter was the only patient in whom anti-ustekinumab antibodies could be detected.

Nine patients underwent IBD-related $(n=8)$ or non-IBD-related $(n=1)$ abdominal surgery within 12 weeks after the previous ustekinumab injection (median 7.7 weeks, IQR 5.6-10.1). Two patients had a surgical site infection and one suffered from a postoperative leakage within the first 30 days postoperatively.

There were 3 pregnancies reported by two women. The first pregnancy occurred (A0P2G3M1) 20 weeks after ustekinumab induction, but ended in a spontaneous abortion 4 weeks after conception. The disease in this female patient was also only moderately controlled. She got pregnant a second time 40 weeks after ustekinumab induction, and administered a last ustekinumab injection at 16 weeks of gestational age. At week 23 of 
pregnancy, she was admitted because of premature contractions, received a cerclage and delivered a healthy boy at week 37 via caesarean section (because of history of perianal CD). The third pregnancy (A0P1G2M1) occurred 16 weeks after ustekinumab induction, and administered a last ustekinumab injection at week 24 of gestational age. The patient received oral corticosteroids at week 34 for a flare of her $C D$ and gave birth to a healthy boy at week 38. No abnormalities have been observed in both babies.

\section{DISCUSSION}

This is the first prospective, real-life cohort of IV-induced ustekinumab-treated CD patients, focusing on serial fCal measurements and endoscopic outcome linked with ustekinumab drug exposure both during induction and during maintenance.

In line with the UNITI post-hoc subgroup analysis which reported endoscopic response and remission rates of $17.4 \%$ and $10.9 \%,{ }^{24}$ we observed an endoscopic response and endoscopic remission rate of $20.5 \%$ and $7.1 \%$, respectively. Although some patients do indeed not achieve the predefined outcome measures due to a mechanistic failure or insufficient drug exposure, the lack of an objective, generally accepted definition of endoscopic remission in CD cannot be disregarded and may contribute to the low endoscopic remission rates reported. ${ }^{28,29}$ The latter may also explain the discrepancy between the higher biological, CRP-defined and lower endoscopic, SES-CD defined, outcome rates after 24 weeks of therapy, as one remaining ulcer with low fCal does not have to imply a poor endoscopic outcome but is not considered endoscopic remission. Prospective attempts to build a novel index of endoscopic remission in ileocolonic CD, including the CREDO project, are therefore eagerly awaited (NCT03498625, NCT03487900). ${ }^{28}$ 
A striking observation was the dynamics in fCal matching the switch from IV induction to SC maintenance therapy. Ustekinumab could induce a significant and clinically relevant decrease in fCal after IV induction in the majority of patients, demonstrating that it is indeed an efficient drug in CD. However, the significant fCal increase beyond week 8 in many patients was associated with disappointing endoscopic outcomes after 6 months. Hence, many of those patients not achieving the primary endpoint should not be considered true primary non-responders due to mechanistic reasons, as they quickly lost their initial response, potentially due to dosing issues. The concept of primary non-response was originally developed to describe patients with lack of clinical improvement following anti-TNF induction doses. This definition has more recently been expanded to also include adequate drug exposure in the definition, besides lack of clinical improvement.

We hypothesise that a too low exposure to ustekinumab (too low induction scheme or too low SC dosage during maintenance) is responsible for the rising fCal levels during maintenance and the low endoscopic remission rates after 6 months. In analogy to infliximab and vedolizumab therapy, more IV doses might be necessary, or in analogy to adalimumab therapy a more frequent SC dosing might be needed to achieve the required exposure to induce endoscopic remission. Indeed, quartile analysis revealed that patients with trough levels within the lowest quartile at several time points, experienced less often a $50 \%$ fCal decrease at week 8 or endoscopic response after 6 months. We identified several informative thresholds with high NPV, implying that patients with serum levels below these targets hardly have a chance to achieve the treatment goals. The UNITI post-hoc analysis identified a maintenance concentration target range from 0.8 to $1.4 \mu \mathrm{g} / \mathrm{mL}$ aiming clinical remission after 6 months. ${ }^{17}$ But, with the ultimate goal of endoscopic remission, higher targets will presumably be needed, as already suggested by our maintenance cut-off of 1.9 
$\mu \mathrm{g} / \mathrm{mL}$ for $-\mathrm{a}$ less stringent - endoscopic response. However, only half of the patients had a maintenance level above $2.1 \mu \mathrm{g} / \mathrm{mL}$, indicating an underexposure in a significant proportion of patients. In a retrospective series, Battat et al. previously suggested an even higher maintenance target of $4.5 \mu \mathrm{g} / \mathrm{mL}$, measured with a different assay. ${ }^{14}$ However, 42 out of their 56 included patients had been dose-escalated to q4w maintenance in order to achieve these high maintenance levels. In contrast to Battat et al. $(25.8 \%),{ }^{14}$ only 2 patients in our cohort were on concomitant immunomodulatory agents. Nevertheless, post-hoc pharmacokinetic data from the UNITI-program did not show better outcomes, nor higher drug levels, for patients receiving combination therapy. ${ }^{17}$

Based on our data and the data available in literature, ${ }^{14,17}$ the Belgian prospective, doubleblinded randomized RESCUE trial will soon be initiated (EudraCT number 2018-004269-14). This study will explore, whether ustekinumab dose-optimisation could improve endoscopic remission rates by rescuing patients who quickly lost response after SC maintenance. Meanwhile, the ongoing STARDUST trial, studying the treat-to-target approach in ustekinumab treated CD patients (NCT03107793), may already inform about the impact of dose-escalation of ustekinumab on endoscopic remission and the ustekinumab serum level target to aim for.

Despite dose-escalation, some patients will inevitable not respond to ustekinumab therapy as was observed earlier with other biological agents. Apart from pharmacokinetics, the underlying biological drivers of the disease in a particular patient contribute to response to therapy, which can be supported by our observation that some patients within the highest quartiles in terms of drug-exposure do not achieve the required endpoints. Similar to the emerging data predicting response to anti-TNF agents, ${ }^{30-33}$ biomarkers predicting response to ustekinumab therapy are urgently needed to identify those patients with the highest likelihood of response based on their underlying biology. 
The prospective nature with standardized clinical, biological and endoscopic assessment, the serial serum level and fCal measurements, the IV induction and the real-life setting are the main strengths of this study. However, we do realize this study has its limitations. First, the majority of the included patients were therapy refractory and failed several biological agents prior to ustekinumab initiation, implying that results may be better in an entirely biologically naive population, as suggested in UNITI-2. Second, although this is the largest real-life study describing the relationship of ustekinumab serum concentration with biomarker and endoscopic outcomes, the sample size remains limited which may result in an underpowered analysis, especially with regard to endoscopic remission. Furthermore, outcomes were limited to 24 weeks. Finally, anti-ustekinumab antibodies were only assessed in samples with an ustekinumab concentration below $1 \mu \mathrm{g} / \mathrm{ml}$, which implies that we could underestimate immunogenicity. Nevertheless, the very low immunogenicity rate even in patients with low ustekinumab serum levels, suggest that the loss-of-response observed in many patients beyond week 8 is not driven by immunogenicity.

In conclusion, ustekinumab is a safe compound, efficacious in inducing clinical and biological remission in refractory $C D$ patients. However, the current $\approx 6 \mathrm{mg}$ per kilogram bodyweight IV induction with subsequent $90 \mathrm{mg}$ SC q8w maintenance dosage results in an insufficient drug exposure in many patients, which ultimately is associated with limited endoscopic remission rates. Prospective randomized trials are warranted to investigate whether we can improve endoscopic remission rates through dose-escalation (qw4) and/or IV re-induction, pro- or reactively. 


\section{TABLES}

Table 1 - Baseline characteristics of all 86 included Crohn's disease patients

Sex, women, $\mathrm{n}(\%)$ $58(67.4)$

Disease duration, $\mathrm{y}$, median (IQR)

$14.7(6.0-23.3)$

Age at initiation of UST, y, median (IQR)

$37.8(28.7-49.3)$

Weighted PRO2, median (IQR)*

$16.0(10.3-21.0)$

- $\quad$ Abdominal pain score, median (IQR)

$1.0(1.0-2.0)$

- $\quad$ Liquid stool frequency, median (IQR)

$4.5(3.0-7.0)$

C-reactive protein, $\mathrm{mg} / \mathrm{L}$, median (IQR)

$12.2(4.5-19.5)$

C-reactive protein > 5mg/L, $\mathrm{n}(\%)$

$60(69.8)$

Faecal calprotectin, $\mu \mathrm{g} / \mathrm{g}$, median (IQR)

$1242.9(470.5-1800.0)$

Serum albumin, g/L, median (IQR)

$40.4(38.0-42.6)$

Simple Endoscopic Score for Crohn's disease, median (IQR)

$12.0(8.0-18.0)$

Body Mass Index, $\mathrm{kg} / \mathrm{m}^{2}$, median (IQR)

$23.2(19.7-26.5)$

Previous biological therapy, n (\%)

- Previous anti-TNF exposure

$82(95.3)$ 


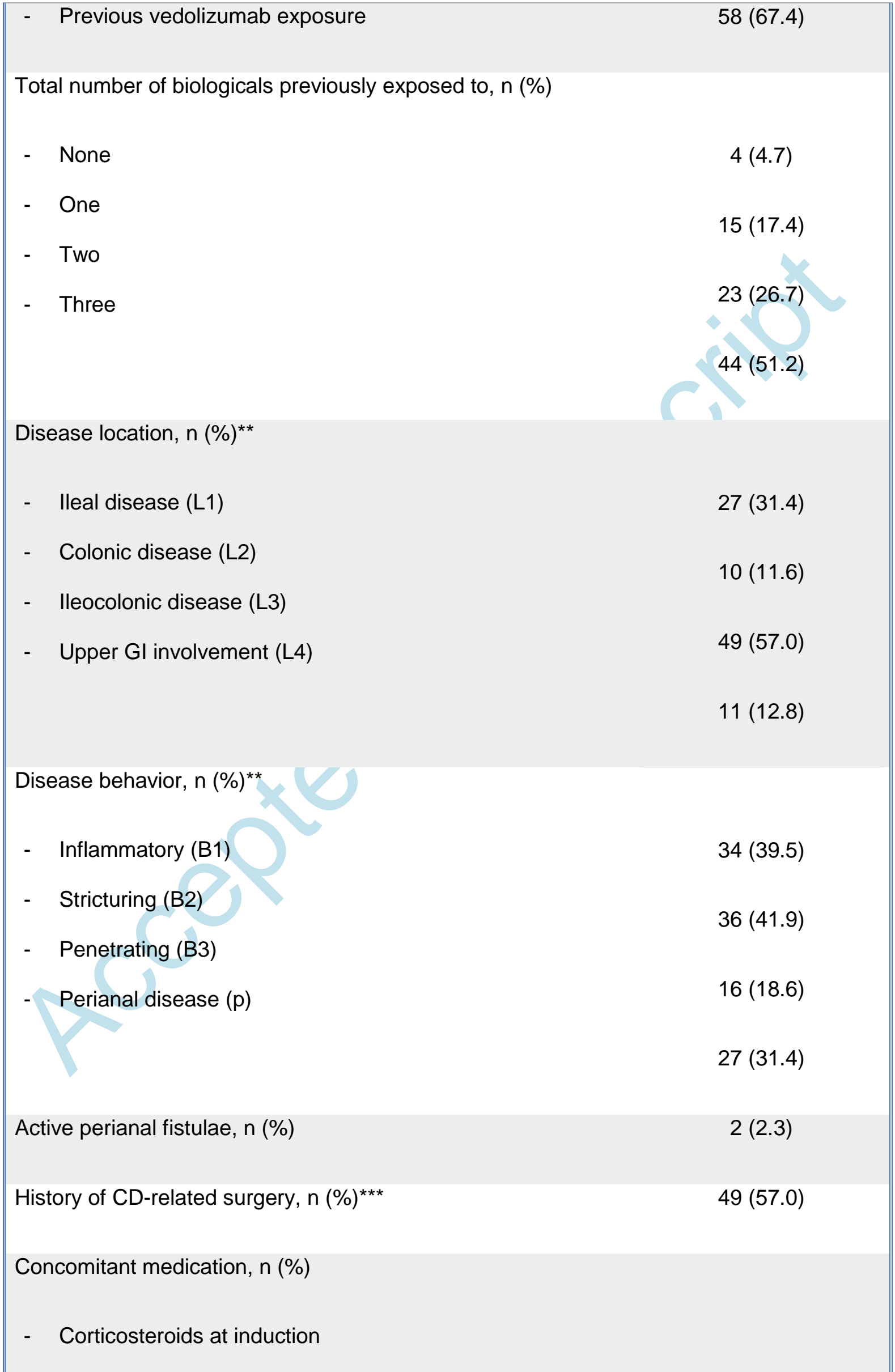


- Topical steroids

- Systemic steroids

$17(19.8)$

- Immunomodulators

- Thiopurines

$13(15.1)$

- Methotrexate

$1(1.2)$

$1(1.2)$

Smoking status, $\mathrm{n}(\%)$

- $\quad$ Active smoking

$20(23.3)$

- Previously smoking

- $\quad$ Never smoked

* Weighted PRO2: 5x abdominal pain score $+2 x$ liquid stool frequency

** Following Montreal classification ${ }^{34}$

*** defined as the occurrence of any resection of a part of the gut, or stricturoplasty for stenosing complications

IQR = interquartile range; $n=$ number of patients; PRO2 = patient reported outcome; TNF = tumour necrosis factor; UST = ustekinumab

1. Silverberg MS, Satsangi J, Ahmad T, et al. Toward an integrated clinical, molecular and serological classification of inflammatory bowel disease: Report of a working party of the 2005 montreal world congress of gastroenterology. Can J Gastroenterol 2005;19 Suppl A:5A-36A. 
Table 2 - Dose-response relationship: defining ustekinumab serum concentration thresholds

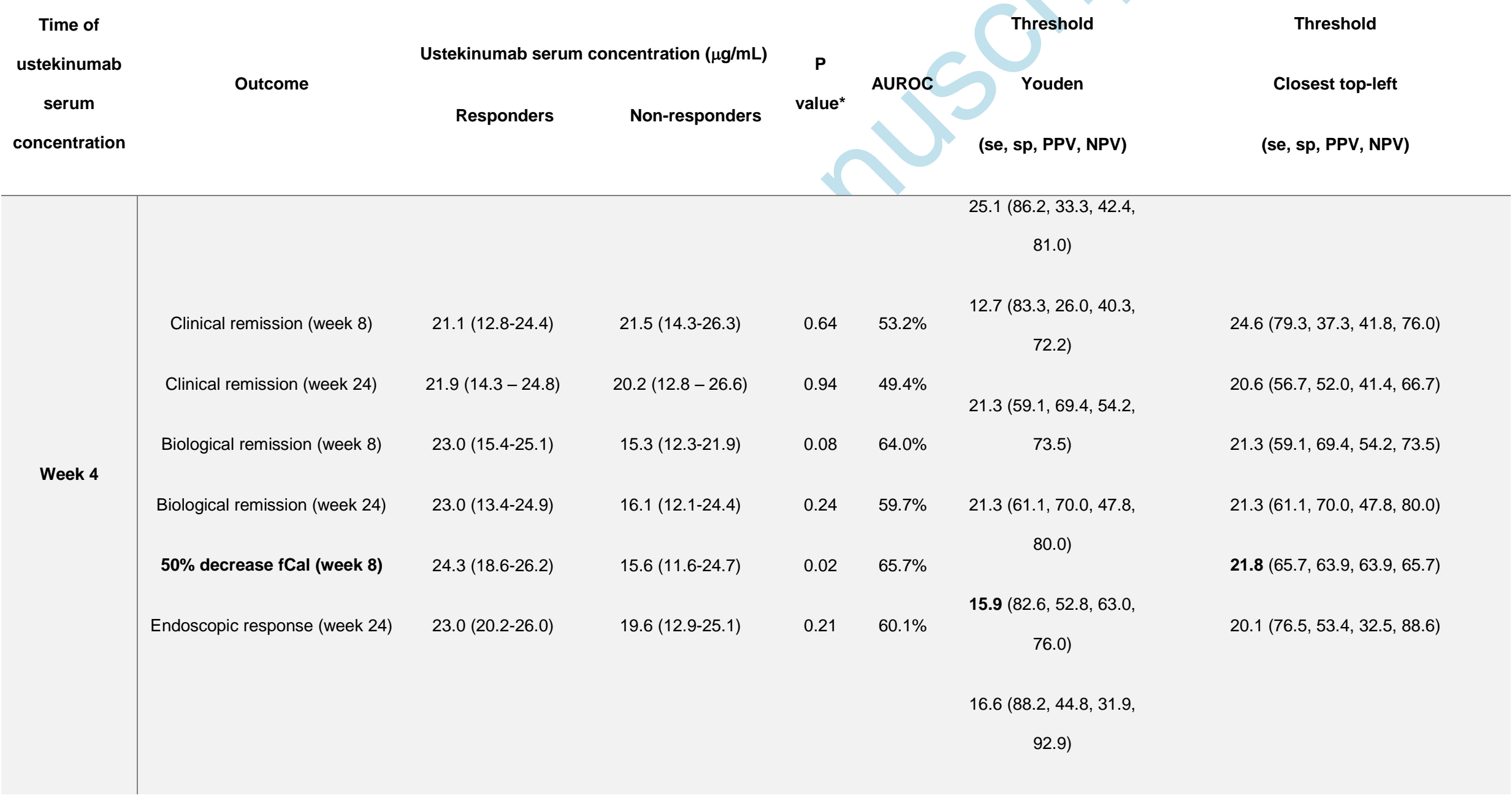


Manuscript Doi: 10.1093/ecco-jcc/jjz008

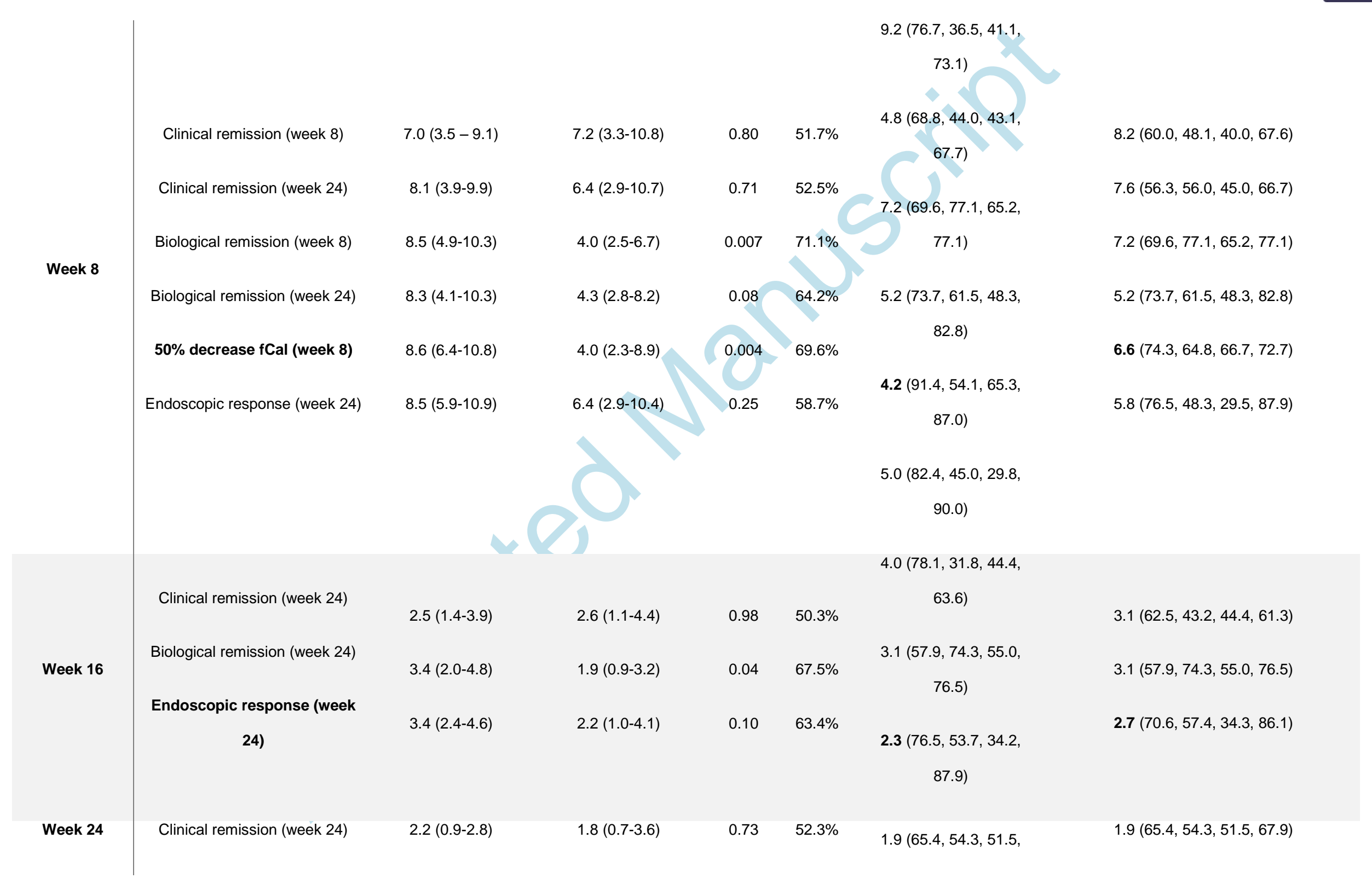


Manuscript Doi: 10.1093/ecco-jcc/jjz008

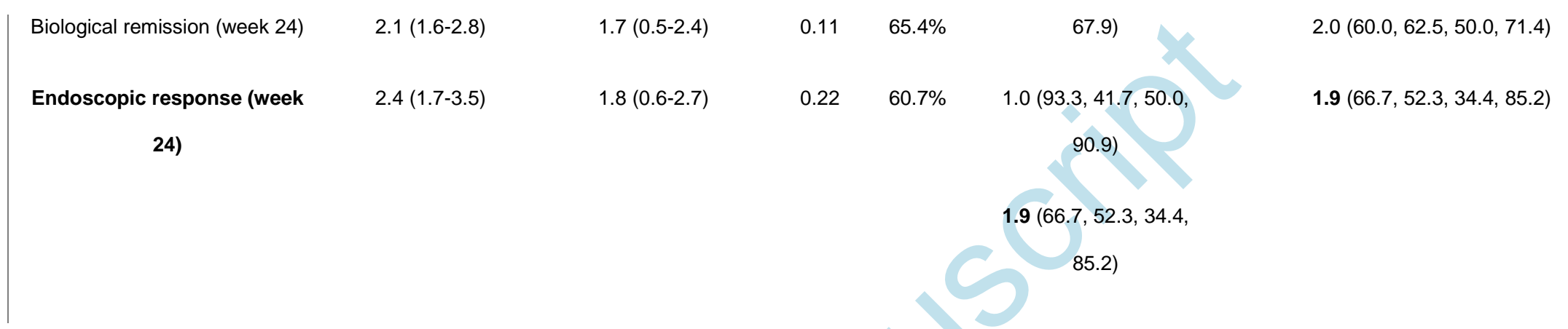

Only exposure-response relations with a p-value <.25 in serum concentrations between responders and nonresponders are depicted. Serum concentrations are reported as median (interquartile range). The threshold in bold is selected based on the response type (endoscopic response > faecal calprotectin > biological remission > clinical remission), the difference in median (interquartile range) serum concentrations of responders and nonresponders (P value, AUROC), se (highest), sp (highest), and se/sp (highest).

AUROC, area under the receiving operating characteristic curve; fCal, faecal calprotectin; NPV, negative predictive value; PPV, positive predictive value; se, sensitivity; sp, specificity.

* Mann-Whitney U test for comparing serum concentrations of responders and nonresponders. Trough concentrations and AUROC are represented as median (interquartile range).

Clinical remission was defined as as an average daily stool frequency $\leq 2.8$ and an average abdominal pain score $\leq 1$. Biological remission was defined as a CRP $\leq 5 \mathrm{mg} / \mathrm{L}$, in patients with an

elevated elevated
CRP at baseline
(CRP
$>5 \mathrm{mg} / \mathrm{L}$ ). Endoscopic

response

was defined

as

a minimal

$50 \%$

decrease

in SES-CD compared

to baseline. 


\section{FIGURE LEGENDS}

Figure 1: Evolution of the Simple Endoscopic Score for Crohn's disease (SES-CD) in all included patients. SES-CD scores are represented as median (interquartile range). ${ }^{* * *} \mathrm{p}<$ 0.001 .

Figure 2: (A) Evolution of C-reactive protein (CRP) in all patients with elevated CRP at baseline $(n=60)$, treated with ustekinumab. CRP is represented as median (interquartile range). (B) Evolution of faecal calprotectin in all included patients with Crohn's disease. Faecal calprotectin levels are represented as median (interquartile range). ${ }^{*} p<0.05,{ }^{* *} p<0.01,{ }^{* * *} p$ $<0.001$.

Figure 3: Quartile analysis depicting the exposure-response relationship between week 4 (A) or week 8 (B) ustekinumab serum levels and a $50 \%$ decrease in faecal calprotectin by week 8 .

Figure 4: Evolution of ustekinumab serum levels over time up to week 24 in all included patients, separated by endoscopic response at week 24. Serum levels are represented as median (interquartile range). No statistical differences between subgroups. 


\section{FUNDING}

B Verstockt is a doctoral fellow and G Van Assche, S Vermeire and M Ferrante are Senior Clinical Investigators of the Research Foundation Flanders (FWO), Belgium. B Verstockt has also received research grants by the Belgium Week of Gastroenterology, the Belgian IBD Research and Development (BIRD), the European Crohn's and Colitis Organization (ECCO) and the IBD Patient's Association Flanders (CCV VZW).

\section{AUTHOR CONTRIBUTIONS}

BV: study design, patient inclusion, data acquisition and interpretation, statistical analysis and drafting of the manuscript. ED: statistical analysis and critical revision of the manuscript. MN, AO, NVDB, IA, GC: data acquisition, critical revision of the manuscript. GVA: patient inclusion, critical revision of the manuscript. AG: data acquisition and interpretation, critical revision of the manuscript. SV and MF: study design, patient inclusion, data interpretation, supervision and critical revision of the manuscript. All authors agreed with the final version of the manuscript.

Guarantor of the manuscript: Marc Ferrante.

\section{CONFLICTS OF INTEREST}

B Verstockt received fianancial support for research from Pfizer; lecture fees from Abbvie, Ferring Pharmaceuticals, Takeda, Rbiopharm and Janssen; consultancy fees from Janssen.

G Van Assche received financial support for research from Abbott and Ferring Pharmaceuticals; lecture fees from Janssen, MSD and Abbott; consultancy fees from PDL BioPharma, UCB Pharma, Sanofi-Aventis, Abbott, Abbvie, Ferring, Novartis, Biogen Idec, Janssen Biologics, NovoNordisk, Zealand Pharma A/S, Millenium/Takeda, Shire, Novartis and Bristol Mayer Squibb.

A Gils received financial support for research from Pfizer, MSD \& Takeda; lecture fees from MSD, Janssen Biologicals, Pfizer, Takeda, Novartis and Abbvie; consultancy fees from Takeda; KU Leuven holds a license agreement with R-biopharm, apDia and Merck.

S Vermeire received financial support for research from MSD, Abbvie, Janssen and UCB Pharma; lecture fees from Abbott, Abbvie, Merck Sharpe \& Dohme, Ferring Pharmaceuticals and UCB Pharma; consultancy fees from Pfizer, Ferring Pharmaceuticals, Shire Pharmaceuticals Group, Merck Sharpe \& Dohme, and AstraZeneca Pharmaceuticals. 
M Ferrante received financial support for research from Takeda and Janssen; lecture fees from Ferring, Boehringer- Ingelheim, Chiesi, Merck Sharpe \& Dohme, Tillotts, Janssen Biologics, Abbvie, Takeda, Mitsubishi Tanabe, Zeria; consultancy fees from Abbvie, Boehringer-Ingelheim, Ferring, Merck Sharpe \& Dohme, and Janssen Biologics.

ED, MN, AO, NVDB, IA, GC declare no conflicts of interest.

The authors have no other relevant affiliations or financial involvement with any organization or entity with a financial interest in or financial conflict with the subject matter or materials discussed in the manuscript apart from those disclose.

\section{ACKNOWLEDGEMENTS}

The authors would like to thank Vera Ballet and Eline Vandeput for an excellent job in maintaining the Leuven IBD patient database; Sophie Organe, Nooshin Ardeshir Davani, Helene Blevi, Tamara Coopmans, Willem-Jan Wollants, Sophie Tops, Els Brouwers and Miet Peeters for an excellent job in maintaining the Biobank database and performing all serum level measurements. 


\section{REFERENCES}

1. Peyrin-Biroulet L, Sandborn W, Sands BE, et al. Selecting therapeutic targets in inflammatory bowel disease (stride): Determining therapeutic goals for treat-to-target. Am J Gastroenterol 2015;110:1324-38.

2. Billiet T, Rutgeerts $P$, Ferrante $M$, Van Assche $G$, Vermeire $S$. Targeting tnf- $\alpha$ for the treatment of inflammatory bowel disease. Expert Opin Biol Ther 2014;14:75-101.

3. Verstockt B, Ferrante M, Vermeire S, Van Assche G. New treatment options for inflammatory bowel diseases. J Gastroenterol 2018;53:585-90.

4. Verstockt B, Van Assche G, Vermeire S, Ferrante M. Biological therapy targeting the il-23/il-17 axis in inflammatory bowel disease. Expert Opin Biol Ther 2017;17:31-47.

5. Leonardi CL, Kimball AB, Papp KA, et al. Efficacy and safety of ustekinumab, a human interleukin-12/23 monoclonal antibody, in patients with psoriasis: 76-week results from a randomised, double-blind, placebo-controlled trial (phoenix 1). Lancet 2008;371:1665-74.

6. Papp KA, Langley RG, Lebwohl M, et al. Efficacy and safety of ustekinumab, a human interleukin-12/23 monoclonal antibody, in patients with psoriasis: 52-week results from a randomised, double-blind, placebo-controlled trial (phoenix 2). Lancet 2008;371:1675-84.

7. Gottlieb A, Menter A, Mendelsohn A, et al. Ustekinumab, a human interleukin $12 / 23$ monoclonal antibody, for psoriatic arthritis: Randomised, double-blind, placebo-controlled, crossover trial. Lancet 2009;373:633-40.

8. Sandborn WJ, Gasink C, Gao LL, et al. Ustekinumab induction and maintenance therapy in refractory crohn's disease. N Engl J Med 2012;367:1519-28.

9. Feagan BG, Sandborn WJ, Gasink C, et al. Ustekinumab as induction and maintenance therapy for crohn's disease. N Engl J Med 2016;375:1946-60.

10. Kopylov U, Afif W, Cohen A, et al. Subcutaneous ustekinumab for the treatment of anti-tnf resistant crohn's disease--the mcgill experience. J Crohns Colitis 2014;8:1516-22.

11. Wils $P$, Bouhnik $Y$, Michetti $P$, et al. Subcutaneous ustekinumab provides clinical benefit for two-thirds of patients with crohn's disease refractory to anti-tumor necrosis factor agents. Clin Gastroenterol Hepatol 2016;14:242-50.e2.

12. Harris KA, Horst S, Gadani A, et al. Patients with refractory crohn's disease successfully treated with ustekinumab. Inflamm Bowel Dis 2016;22:397-401.

13. Ma C, Fedorak RN, Kaplan GG, et al. Clinical, endoscopic and radiographic outcomes with ustekinumab in medically-refractory crohn's disease: Real world experience from a multicentre cohort. Aliment Pharmacol Ther 2017. 
14. Battat R, Kopylov U, Bessissow T, et al. Association between ustekinumab trough concentrations and clinical, biomarker, and endoscopic outcomes in patients with crohn's disease. Clin Gastroenterol Hepatol 2017;15:1427-34 e2.

15. Detrez I, Van Stappen T, Martin Arranz MD, Papamichael K, Gils A. Current practice for therapeutic drug monitoring of biopharmaceuticals in inflammatory bowel disease. Ther Drug Monit 2017;39:344-9.

16. Feuerstein JD, Nguyen GC, Kupfer SS, et al. American gastroenterological association institute guideline on therapeutic drug monitoring in inflammatory bowel disease. Gastroenterology 2017;153:827-34.

17. Adedokun OJ, Xu Z, Gasink C, et al. Pharmacokinetics and exposure response relationships of ustekinumab in patients with crohn's disease. Gastroenterology 2018;154:1660-71.

18. Khorrami S, Ginard D, Marin-Jimenez I, et al. Ustekinumab for the treatment of refractory crohn's disease: The spanish experience in a large multicentre open-label cohort. Inflamm Bowel Dis 2016;22:1662-9.

19. Claire P, Severine B, Nicolas D, et al. P360 trough levels and antibodies to ustekinumab are not correlated to response to ustekinumab treatment in crohn's disease patients. 12th Congress of ECCO Barcelona, 2017.

20. Khanna R, Zou G, D'Haens G, et al. A retrospective analysis: The development of patient reported outcome measures for the assessment of crohn's disease activity. Aliment Pharmacol Ther 2015;41:77-86.

21. Feagan BG, Sandborn WJ, D'Haens G, et al. Induction therapy with the selective interleukin-23 inhibitor risankizumab in patients with moderate-to-severe crohn's disease: A randomised, double-blind, placebo-controlled phase 2 study. Lancet 2017;389:1699-709.

22. Sturm A, Maaser C, Calabrese E, et al. Ecco-esgar guideline for diagnostic assessment in inflammatory bowel disease. J Crohns Colitis 2018.

23. Ferrante $\mathrm{M}$, Colombel JF, Sandborn WJ, et al. Validation of endoscopic activity scores in patients with crohn's disease based on a post hoc analysis of data from sonic. Gastroenterology 2013;145:978-86 e5.

24. Rutgeerts P, Gasink C, Chan D, et al. Efficacy of ustekinumab in inducing endoscopic healing in patients with crohn's disease. Gastroenterology 2018.

25. Marini JC, Gils A, Shankar G, et al. P649 comparison of the ku leuven ustekinumab concentration assay and the antibodies-to-ustekinumab assay with assays developed at janssen $r \& d$ and used in clinical studies of ibd patients. 13th Congress of ECCO Vienna, 2018.

26. Van Stappen T, Bollen L, Vande Casteele N, et al. Rapid test for infliximab drug concentration allows immediate dose adaptation. Clin Trans/ Gastroenterol 2016;7:e206. 
27. Verstockt B, Deleenheer B, Van Assche G, Vermeire S, Ferrante M. A safety assessment of biological therapies targeting the il-23/il-17 axis in inflammatory bowel diseases. Expert Opin Drug Saf 2017;16:809-21.

28. Bossuyt P, Louis E, Mary JY, Vermeire S, Bouhnik Y. Viewpoint: Defining endoscopic remission in ileocolonic crohn's disease: Let's start from scratch. J Crohns Colitis 2018.

29. Maaser C, Sturm A, Vavricka SR, et al. Ecco-esgar guideline for diagnostic assessment in inflammatory bowel disease. J Crohns Colitis 2018.

30. West NR, Hegazy AN, Owens BMJ, et al. Oncostatin $m$ drives intestinal inflammation and predicts response to tumor necrosis factor-neutralizing therapy in patients with inflammatory bowel disease. Nat Med 2017;23:579-89.

31. Gaujoux R, Starosvetsky E, Maimon N, et al. Cell-centred meta-analysis reveals baseline predictors of anti-tnfalpha non-response in biopsy and blood of patients with ibd. Gut 2018.

32. Verstockt B, Verstockt S, Blevi H, et al. Trem-1, the ideal predictive biomarker for endoscopic healing in anti-tnf-treated crohn's disease patients? Gut 2018.

33. Verstockt B, Verstockt S, Creyns B, et al. Mucosal il13ra2 expression predicts non-response to anti-tnf therapy in crohn's disease. Aliment Pharmacol Ther 2018.

34. Silverberg MS, Satsangi J, Ahmad T, et al. Toward an integrated clinical, molecular and serological classification of inflammatory bowel disease: Report of a working party of the 2005 montreal world congress of gastroenterology. Can J Gastroenterol 2005;19 Suppl A:5A-36A.

Figure 1 
Manuscript Doi: 10.1093/ecco-jcc/jjz008

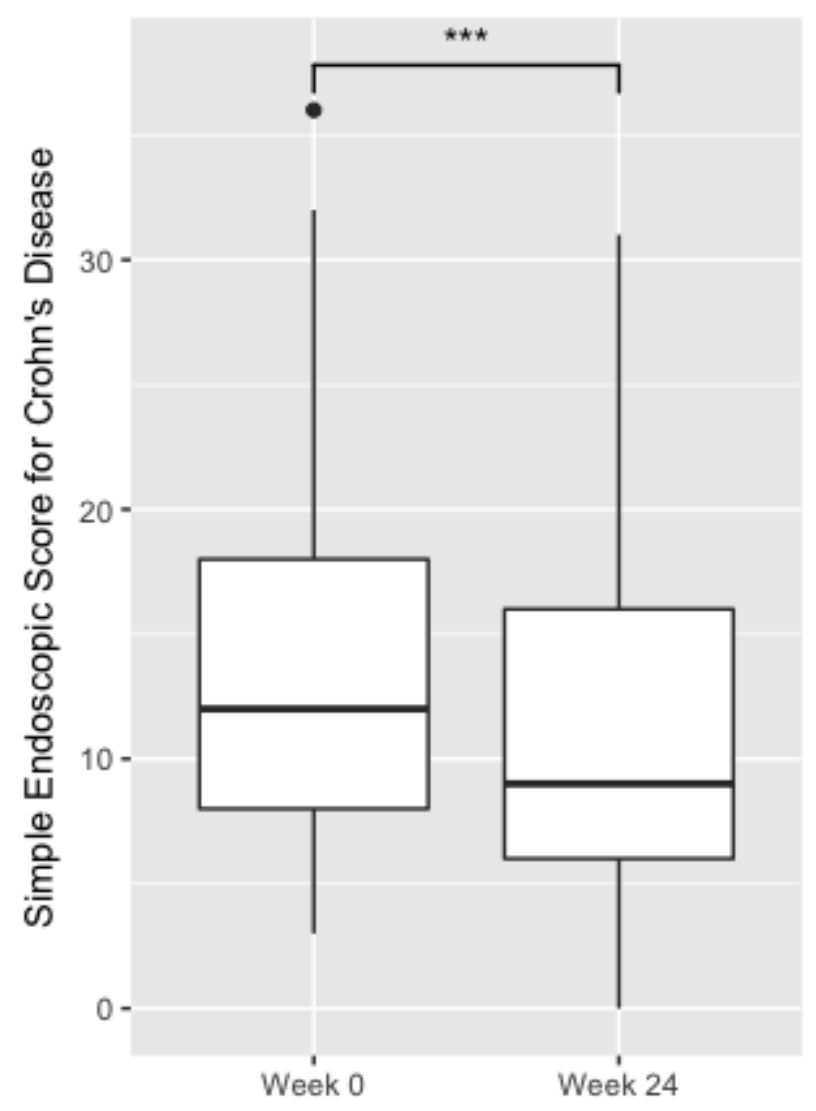

Follow-up (weeks) 
Figure 2

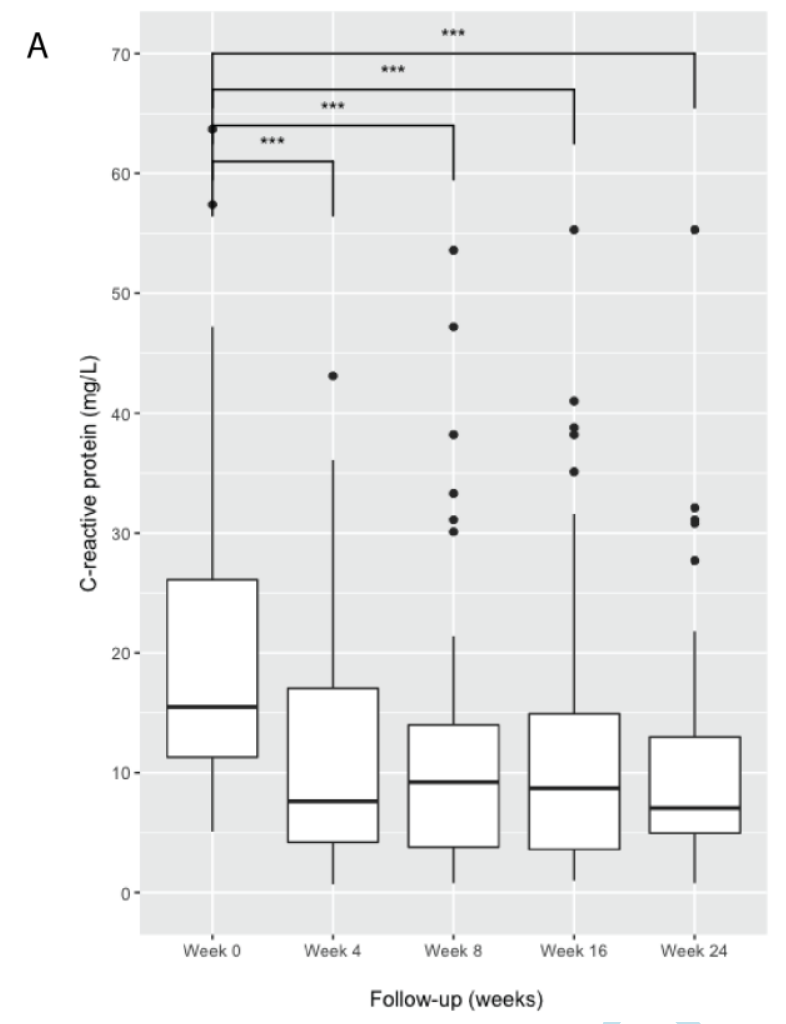

B

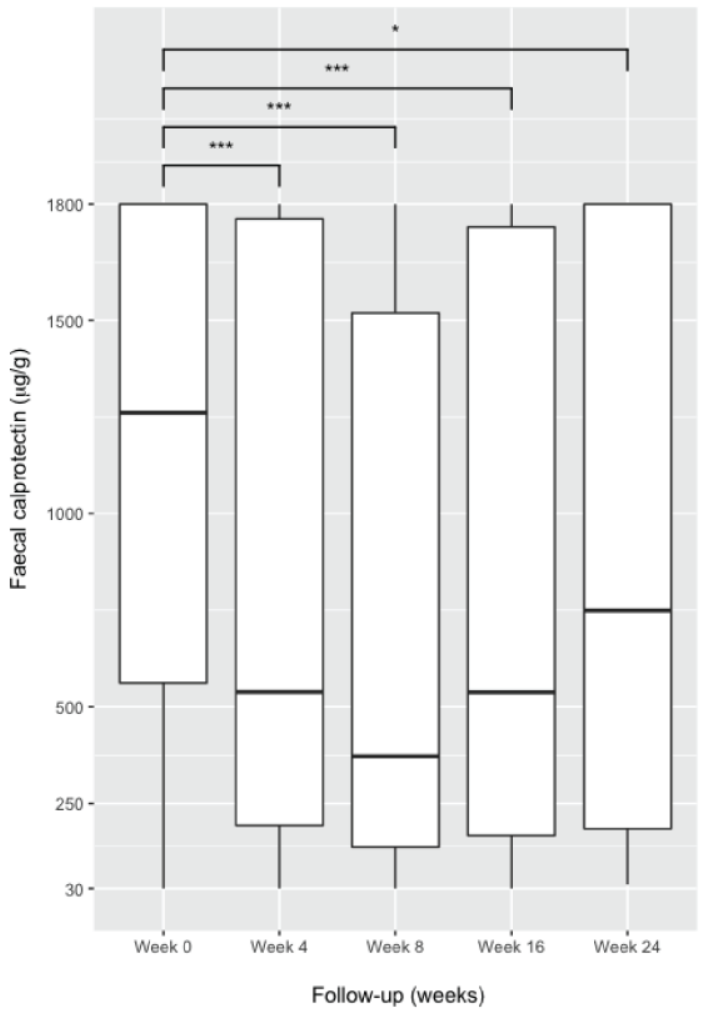


Figure 3
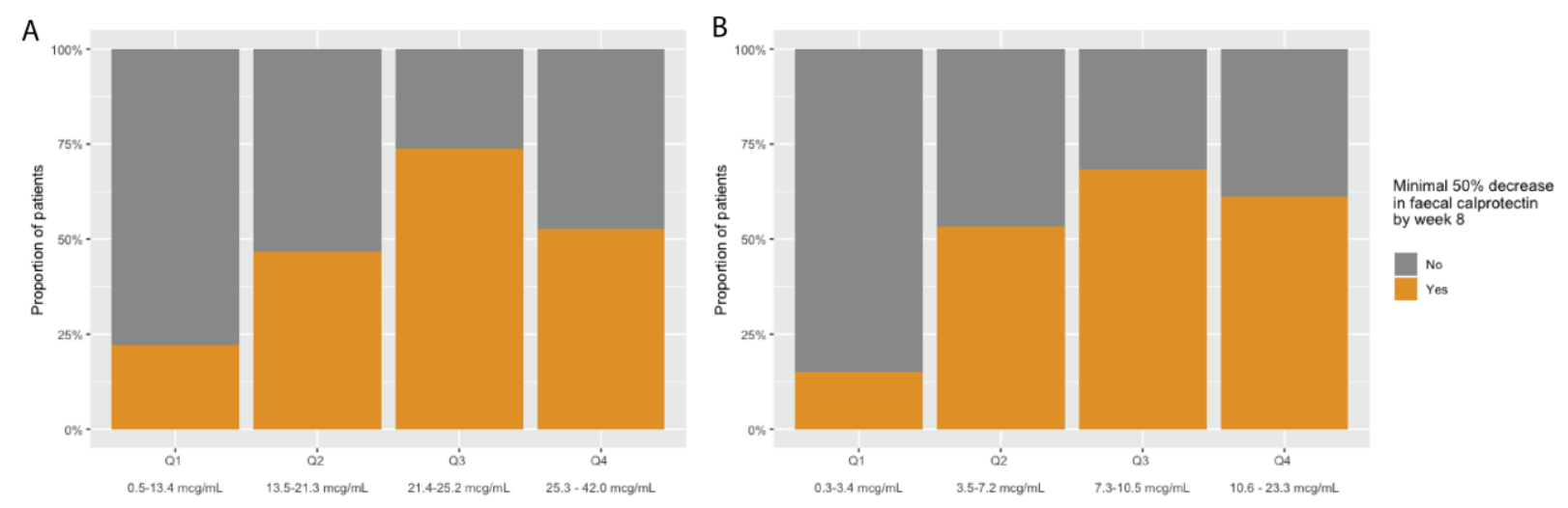
Figure 4

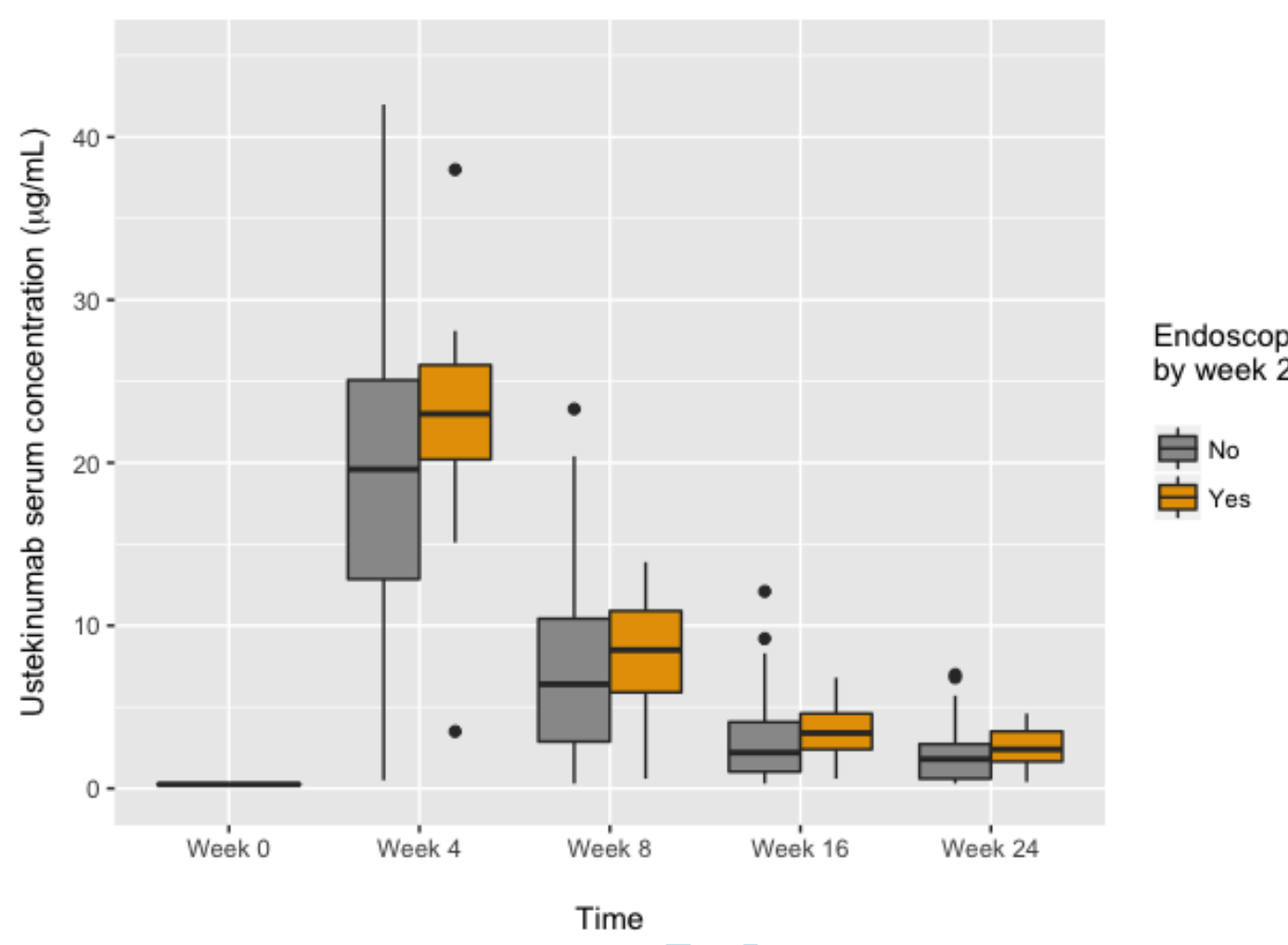

